\title{
RESEÑA BIBLIOGRÁFICA
}

MOSCOSO, N. (compiladora). Estadías Hospitalarias Inadecuadas: Aspectos Metodológicos para su Detección, Estimación y Predicción. Ediuns, Bahía Blanca, 2015, 104 pp.

El libro Estadias hospitalarias inadecuadas: Aspectos metodológicos para su detección, estimación y predicción reúne cinco trabajos de un grupo interdisciplinario de docentes e investigadores de Medicina, Economía y Gestión de los Servicios de Salud de la Universidad Nacional del Sur, así como a profesionales de Medicina, Administración e Informática del Hospital Municipal de Agudos Dr. Leónidas Lucero de la ciudad de Bahía Blanca. Los trabajos presentados en el libro tienen como eje transversal la conceptualización y medición de los denominados dias de estada inadecuados, que son definidos como aquellos en los que un paciente que fue internado podría haber sido atendido en centros asistenciales de diferente complejidad, o en menor tiempo; o cuando directamente no debió estar internado. El libro se divide en cinco capítulos.

El primer capítulo desarrolla el marco conceptual que da sustento al resto de los trabajos. Se caracteriza al hospital como organización productiva, destacando las complejidades inherentes a la medición de su output. En particular, cada producto (o paciente) es diferente al resto, en el sentido que requiere de un proceso de atención personalizado que varía según factores propios del individuo, del profesional que lo asiste y de las tecnologías disponibles. Las dificultades para medir el producto hospitalario se traducen en problemas para evaluar la eficiencia (tanto técnica como económica) de este tipo de organizaciones, dado que dicha evaluación requiere comparar el uso de recursos con la cantidad y calidad de los productos elaborados. Sin embargo, en la práctica existe consenso en utilizar como medida de producto la duración de la estancia (entendida como la diferencia en días entre la fecha de alta e ingreso al establecimiento) y como indicador de eficiencia del insumo cama a la estancia media (estándar o corregida) que cuantifica el número de días de internación promedio por paciente egresado de un servicio u hospital.

En el capítulo dos los autores analizan distintos indicadores de gestión hospitalaria. En relación con el indicador de "estancia media", se exponen sus falencias para evaluar la eficiencia, señalando que i) no discrimina entre casos según su complejidad, pues le asigna a todos los pacientes atendidos el mismo peso relativo en la cuantificación de la producción (días de estada) independientemente 
del consumo de recursos (por ejemplo, medicamentos o insumos descartables) y ii) el promedio resultante no se expone depurado de aquellas situaciones poco frecuentes y con valores extremos que prolongan las internaciones por encima de la media normal de cada patología o servicio. Estos casos, conocidos en la literatura como "outliers", se asocian a internaciones que por diversas razones superan el consumo de recursos y duración promedio de las hospitalizaciones.

Sobre la base de una revisión exhaustiva de la literatura, en el capítulo tres se estudia cuáles son las causas que pueden derivar en la aparición de días de estancia inadecuados, clasificándolas en cuatro grupos: i) inherentes a problemas en la planificación y gestión del hospital (por ejemplo, demoras en la realización de alguna prueba diagnóstica), ii) vinculadas a decisiones del personal médico (demoras en firmar un alta, por ejemplo), iii) bajo responsabilidad del paciente y sus familiares (como retardos en la adaptación del hogar para recibir al paciente externado) y iv) atribuibles a la gestión del sistema de salud (por ejemplo, retrasos en la autorización de ciertos procedimientos médicos). La utilidad de esta clasificación es que cada grupo de causales requerirá de distintas acciones para su eliminación o reducción.

En el capítulo cuatro se resumen algunas investigaciones que revelan el uso inadecuado del recurso cama en la Argentina. Al evaluar la adecuación de los ingresos y estancias en los pacientes egresados durante 2001 en un hospital de agudos de la Ciudad Autónoma de Buenos Aires, se concluyó que el 15\% de los ingresos y entre el $30-40 \%$ de los días de internación resultaron inadecuados. En el mismo capítulo, se presenta la evaluación de los días de internación en un hospital público de jurisdicción municipal de la ciudad de Bahía Blanca (provincia de Buenos Aires) donde se muestra que aproximadamente el 12\% del total resultaron inadecuados. En este último caso, las principales causas de inadecuación fueron la presencia de pacientes en condiciones de vulnerabilidad social y las demoras en trámites atribuibles a los seguros de salud.

Por último, en el capítulo cinco se desarrollan las herramientas estadísticas que permiten analizar el comportamiento de la variable "día de estada". En particular, se sostiene la utilidad de implementar métodos estadísticos multivariados para pronosticar su valor en los distintos pacientes, a partir de la selección de predictores.

En los sistemas de salud, el uso inadecuado del recurso cama produce efectos económicos adversos, por lo cual adquiere relevancia implementar mecanismos que permitan reconocerlo y minimizarlo. Esto es especialmente relevante en 
el caso de los hospitales del subsector público, que deben atender las necesidades de cuidados médicos de los segmentos más vulnerables de la sociedad. En este sentido, el libro Estadías hospitalarias inadecuadas: Aspectos metodológicos para su detección, estimación y predicción realiza un aporte de suma importancia, al sistematizar en un mismo volumen un marco conceptual y las técnicas estadísticas necesarias para el análisis e identificación del fenómeno de las instancias inadecuadas. Los resultados expuestos sin duda habrán de constituir una herramienta clave para la mejora de la gestión hospitalaria, y para el diseño de intervenciones que se propongan reducir las ineficiencias en el uso de los recursos asignados al cuidado de la salud.

Fernando Lago 
